\title{
Pulsar Velocities without Neutrino Mass
}

astro-ph/9803002

\author{
D. Grasso ${ }^{1}$, H. Nunokawa ${ }^{2}$ and J. W. F. Valle ${ }^{1}$ \\ ${ }^{1}$ Instituto de Física Corpuscular - IFIC/CSIC, Departamento de Fúsica Teòrica \\ Universitat de València, 46100 Burjassot, València, Spain \\ ${ }^{2}$ Instituto de Física Gleb Wataghin, Universidade Estadual de Campinas \\ 13083-970 Campinas, São Paulo, Brazil
}

(July 1, 2018)

\begin{abstract}
We show that pulsar velocities may arise from anisotropic neutrino emission induced by resonant conversions of massless neutrinos in the presence of a strong magnetic field. The main ingredient is a small violation of weak universality and neither neutrino masses nor magnetic moments are required.
\end{abstract}

One of the most challenging problems in modern astrophysics is to find a consistent explanation for the high velocity of pulsars. Observations [1] show that these velocities range from zero up to $900 \mathrm{~km} / \mathrm{s}$ with a mean value of $450 \pm 50 \mathrm{~km} / \mathrm{s}$. An attractive possibility is that pulsar motion arises from an asymmetric neutrino emission during the supernova (SN) explosion. In fact, neutrinos carry more than $99 \%$ of the new-born protoneutron star's gravitational binding energy so that even a $1 \%$ asymmetry in the neutrino emission could generate the observed pulsar velocities. To find the origin of such asymmetry is, however, not a minor task. One intriguing possible explanation to this puzzle may reside in the interplay between the parity non-conservation present in weak interactions and the strong magnetic fields which are expected during a SN explosion. Although several possible realizations of this idea in the framework of the Standard Model (SM) of particle physics have been already explored [2,3] a conclusive solution of the problem is still lacking, and there is some motivation for looking also at solutions that involve physics beyond the SM.

Recently, several neutrino conversion mechanisms in matter have been invoked as a possible engine for powering pulsar motion. Kusenko and Segré proposed a mechanism [4] based on MSW conversions [5]. The idea is based on the observation that the strong magnetic field present during a SN explosion gives rise to some angular dependence of the matter induced neutrino potentials [6]. As a consequence, in the presence of non-vanishing $\nu_{\tau}$ mass and mixing the resonance sphere for the $\nu_{e}-\nu_{\tau}$ conversions is distorted. If the resonance surface lies between the $\nu_{\tau}$ and $\nu_{e}$ neutrino spheres, such a distortion would induce a temperature anisotropy in the flux of the escaping tau-neutrinos produced by the conversions, hence a recoil kick of the proto-neutron star. In order to account for the observed pulsar velocities the required strength of the dipolar component of the magnetic field between the two neutrino spheres must exceed $10^{15}$ Gauss [7] or even larger 87. Another crucial ingredient in this mechanism is the neutrino squared mass difference, $\Delta m^{2} \gtrsim 10^{4} \mathrm{eV}^{2}$, which leads to $m_{\nu_{\tau}} \gtrsim 100 \mathrm{eV}$ or so, assuming a negligible $\nu_{e}$ mass. This is necessary in order for the resonance surface to be located between the two neutrino-spheres. It should be noted, however, that such requirement is at odds with cosmological bounds on neutrinos masses unless the $\tau$-neutrino is unstable.

Akhmedov, Lanza and Sciama [9] proposed a similar pulsar acceleration mechanism based on resonant neutrino spin-flavour precession (RSFP) [10]. The magnetic field not only affects the medium properties, as in the matter density in the MSW case, but also induces the spin-flavour precession through its coupling to the neutrino transition magnetic moment [11]. The lowest magnetic field strength required is $B>2 \times 10^{16}$ Gauss, as long as the neutrino magnetic moment exceeds $\mu_{\nu} \gtrsim 10^{-15} \mu_{B}$.

In this letter we investigate the relevance, for pulsar motion, of a different kind of neutrino conversions not requiring any neutrino mass nor magnetic moment. The basic mechanism was proposed over ten years ago in ref. [12. In contrast to the physics of the solar neutrino problem, the new mechanism was shown to be potentially relevant for supernova physics. This has been recently studied in more detail in ref. [13] where stringent limits have been derived.

The simplest underlying particle physics model that realizes this new conversion mechanism postulates the existence of two new $S U(2) \otimes U(1)$ singlet leptons for each generation of leptons, in such a way that lepton number symmetry is exact in the Lagrangian [14]. These extra states can arise in various extensions of the SM, such as superstring models 15. However, the model is very interesting on its own right, both conceptually as well as phenomenologically [16]. As a result of the postulated lepton number symmetry neutrinos remain massless to all orders of perturbation even after the gauge 
symmetry breaking. However, unlike the situation in the SM there is a non-trivial Kobayashi-Maskawa-like mixing in the weak leptonic charged current [17]. The simplest such scheme contains three two-component gauge singlet neutral leptons $S$ added to the three right-handed neutrino components $\nu^{c}$ present in $\mathrm{SO}(10)$. For definiteness we consider this model at the $S U(2) \otimes U(1)$ level. The assumed conservation of lepton number leads to a neutral mass matrix with the following texture in the basis $\left(\nu, \nu^{c}, S\right)$ :

$$
\left(\begin{array}{ccc}
0 & D & 0 \\
D^{T} & 0 & M \\
0 & M^{T} & 0
\end{array}\right),
$$

where the Dirac matrix $D$ describes the coupling between the weak doublet $\nu$ and the singlet $\nu^{c}$, and whereas $M$ connects the singlet states $\nu^{c}$ and $S$. It is easy to see that, as expected, the three conventional neutrinos remain massless, while the other six neutral 2-component leptons combine into three heavy Dirac fermions. This model offers a viable alternative to the see-saw model.

The phenomenological implications of this picture are manifest when considering the resulting charged-current Lagrangian in the massless-neutrino sector:

$$
\mathcal{L}_{\mathrm{CC}}=\frac{\mathrm{i} g}{\sqrt{2}} W_{\mu} \bar{e}_{a L} \gamma_{\mu} K_{a i} \nu_{i L}+\text { h.c. },
$$

where $a=e, \mu, \tau, i=1,2,3$ and the mixing matrix $K$ is given as 12,17.

$$
K=R \mathcal{N}
$$

For definiteness and simplicity we will confine ourselves to the case of two neutrinos. In this case $R$ is a $2 \times 2$ rotation matrix,

$$
R=\left(\begin{array}{cc}
\cos \theta & \sin \theta \\
-\sin \theta & \cos \theta
\end{array}\right)
$$

and where the diagonal matrix,

$$
\mathcal{N}=\left(\begin{array}{cc}
\mathcal{N}_{1} & 0 \\
0 & \mathcal{N}_{2,3}
\end{array}\right)
$$

Note that $K$ is not unitary, since it is a sub-matrix of the full rectangular matrix including also the heavy states [17]. The matrix $\mathcal{N}$ describes the effective non-orthogonality of the two neutrino flavours, i.e., $\left\langle\nu_{e} \mid \nu_{\mu, \tau}\right\rangle \equiv-\sin \theta \cos \theta\left(\mathcal{N}_{1}^{2}-\mathcal{N}_{2,3}^{2}\right)$. The non-diagonal elements of the matrix $K$ cannot be rotated away through a redefinition of the massless-neutrino fields. In this way a non-trivial mixing arises among the massless neutrinos.

The corresponding form of the neutral-current Lagrangian for the massless-neutrino sector is 12,17]

$$
\mathcal{L}_{\mathrm{NC}}=\frac{\mathrm{i} g}{2 \cos \theta_{W}} Z_{\mu} P_{i j} \bar{\nu}_{i L} \gamma_{\mu} \nu_{j L}
$$

where

$$
P=K^{\dagger} K=\mathcal{N}^{2}
$$

The matrix $P$ is diagonal but generation-dependent, signalling the violation of weak universality. It is also convenient to define

$$
\mathcal{N}_{i}^{2} \equiv\left(1+h_{i}^{2}\right)^{-1}, \quad i=1,2(3),
$$

where the $h_{i}$ parameters reflect the deviation from the standard neutrino coupling. Since no oscillations between two strictly massless neutrinos can develop in vacuum, it follows that laboratory limits on the leptonic mixing angle $\theta$ are very weak. However, it will be sufficient for our purposes to assume that the mixing angle $\theta$ is very small. In this way we have $\nu_{i} \sim \nu_{a}[a=e, \mu(\tau)]$, so that $h_{i}^{2} \sim h_{a}^{2}$. The parameters $h_{i}^{2}$ are also constrained experimentally. There have been extensive experimental studies of the constraints on $h_{a}^{2}$. For the third generation one can still allow $h_{\tau}^{2}$ values in the range of a few percent [18], whereas the constraints on $h_{e}^{2}$ and $h_{\mu}^{2}$ are more stringent. For this reason we consider from now on the case of $\nu_{e} \nu_{\tau}$ conversions.

In the flavour eigenstate-type basis $\tilde{\nu}_{a}$ defined in ref. 12], the Schroedinger evolution equation describing the neutrino propagation in matter can be written as

$$
i \frac{d}{d r}\left(\begin{array}{c}
\tilde{A}_{e} \\
\tilde{A}_{\tau}
\end{array}\right)=\sqrt{2} G_{F} \frac{\rho}{m_{N}}\left(\begin{array}{cc}
\tilde{H}_{e} & \tilde{H}_{e \tau} \\
\tilde{H}_{e \tau} & \tilde{H}_{\tau}
\end{array}\right)\left(\begin{array}{c}
\tilde{A}_{e} \\
\tilde{A}_{\tau}
\end{array}\right),
$$

where $\tilde{A}_{e, \tau}$ are the neutrino amplitudes, $G_{F}$ is the Fermi constant, $\rho$ is the matter density, and $m_{N}$ is the nucleon mass. The entries of the evolution Hamiltonian are now given by 12,13

$$
\begin{aligned}
\tilde{H}_{e} & =Y_{e}^{\prime}\left(\mathcal{N}_{e} c^{2}+\mathcal{N}_{\tau} s^{2}\right)^{2}-\frac{1}{2} Y_{n}^{\prime}\left(\mathcal{N}_{e}^{2} c^{2}+\mathcal{N}_{\tau}^{2} s^{2}\right), \\
\tilde{H}_{e \tau} & =\left[Y_{e}^{\prime}\left(\mathcal{N}_{e} c^{2}+\mathcal{N}_{\tau} s^{2}\right)-\frac{1}{2} Y_{n}^{\prime}\left(\mathcal{N}_{e}+\mathcal{N}_{\tau}\right)\right]\left(\mathcal{N}_{\tau}-\mathcal{N}_{e}\right) s c \\
\tilde{H}_{\tau} & =Y_{e}^{\prime} s^{2} c^{2}\left(\mathcal{N}_{\tau}^{2}-\mathcal{N}_{e}^{2}\right)-\frac{1}{2} Y_{n}^{\prime}\left(\mathcal{N}_{e}^{2} s^{2}+\mathcal{N}_{\tau}^{2} c^{2}\right)
\end{aligned}
$$

where

$$
Y_{e}^{\prime} \equiv Y_{e}-Y_{e}^{0} \cos \phi \quad Y_{n}^{\prime} \equiv Y_{n}+Y_{e}^{0} \cos \phi
$$

where $Y_{e} \equiv \frac{n_{p}}{n_{p}+n_{n}}$ and the superscript zero refers to the contribution from the lowest Landau level 19]. In eq. (10) we used the shorthand notation $s \equiv \sin \theta$ and $c \equiv \cos \theta$. The effect of the magnetic field enters through the neutrino potentials [6] that affect the entries of the Hamiltonian. This can be parametrized by defining effective electron and neutron fractions $Y_{e}^{\prime}$ and $Y_{n}^{\prime}$ which now contain an anisotropic term $\cos \phi$ where $\phi$ is the angle between the neutrino propagation direction and $\vec{B}$. Using eq. (10) the resonance condition, $\tilde{V}_{e}=\tilde{V}_{\tau}$, reads 


$$
Y_{e}^{\prime}=\eta Y_{n}^{\prime}
$$

or, in a more transparent form,

$$
Y_{e}\left(1+\lambda_{e} \cos \phi\right)=\eta Y_{n}\left(1-\lambda_{e} \frac{Y_{e}}{Y_{n}} \cos \phi\right) .
$$

where $\lambda_{e} \equiv n_{e}^{0} / n_{e}$ where the superscript zero again the contribution to the electron fraction coming from the lowest Landau level. The parameter $\eta$ defined as

$$
\eta \equiv \frac{1}{2}\left(h_{\tau}^{2}-h_{e}^{2}\right)
$$

measures the deviation from lepton universality which, for the $\left(\nu_{e}, \nu_{\tau}\right)$ system may reach $\eta \sim 10^{-2}$ or so [18].

In order to establish that massless neutrino conversions can play a role explaining the origin of pulsar velocities, we need to verify that the resonance condition eq. (12) can indeed be fulfilled in a SN environment between the $e$ and the $\tau$ neutrino-spheres. The mean resonance position is obtained by averaging eq. (13) over $\phi$, giving

$$
Y_{e}=\eta Y_{n} .
$$

Apart from small $\mathbf{B}$ induced corrections to $Y_{e}$, the condition eq. (15) coincides with the free-field resonance condition given in ref. 13] which allows us to apply here some of the arguments used there. As $Y_{n} \approx 1$ in the core of the SN, we see that condition in eq. (15) can be fulfilled for experimentally allowed $\eta$ values if $Y_{e} \lesssim 10^{-2}$. This is indeed possible close to the neutrino-spheres as a consequence of the strong deleptonization taking place in that region during the Kelvin-Helmholtz cooling phase. A rough estimate of the value of $Y_{e}$ between the neutrinospheres can obtained following ref. [13]. From the approximate chemical equilibrium for $e^{-}, p, n$, and $\nu_{e}$, we have $\mu_{e^{-}}+\mu_{p} \sim \mu_{n}$, where $\mu_{e^{-}}$is the electron chemical potential and we have set $\mu_{\nu_{e}} \sim 0$. Using Boltzmann statistics for non-relativistic nucleons and the above chemical equilibrium condition, we can write

$$
Y_{e} \sim \frac{1}{\exp \left(\mu_{e^{-}} / T\right)+1},
$$

neglecting the neutron-proton mass difference.

The chemical potential for relativistic and degenerate electrons near the neutrinosphere is given by

$$
\mu_{e^{-}} \approx\left(3 \pi^{2} n_{e}\right)^{1 / 3} \approx 51.6\left(Y_{e} \rho_{12}\right)^{1 / 3} \mathrm{MeV},
$$

where $\rho_{12}$ is the matter density in units of $10^{12} \mathrm{~g} \mathrm{~cm}^{-3}$. For typical conditions between the two neutrino-spheres, $T \sim 4 \mathrm{MeV}$ and $\rho_{12} \sim 1 \div 10$ we find $Y_{e} \sim 5 \times 10^{-2} \div$ $5 \times 10^{-3}$. These analytical results are in good agreement with numerical SN models. Therefore, we can expect resonant massless-neutrino conversion to occur between the two neutrino-spheres for a range of values of the parameter $\eta$ which is not experimentally excluded.
The adiabaticity of resonant massless-neutrino conversions can be easily verified by looking at the probability for $\nu_{e} \leftrightarrow \nu_{\tau}$ and $\bar{\nu}_{e} \leftrightarrow \bar{\nu}_{\tau}$ conversions, given by [13]

$$
\begin{aligned}
P & =1-\exp \left(-\frac{\pi^{2}}{2} \frac{\delta r}{L_{m}^{0}}\right) \\
& \approx 1-\exp \left[-32 \rho_{12}\left(r_{0}\right)\left(\frac{\eta}{10^{-2}}\right)\left(\frac{h_{Y_{e}}\left(r_{0}\right)}{1 \mathrm{~cm}}\right) \sin ^{2} 2 \theta\right] .
\end{aligned}
$$

Resonant conversion is adiabatic if $P \simeq 1$, hence whenever the resonance width $\delta r=2 h_{Y_{e}} \sin 2 \theta$ is larger than the conversion length at resonance $L_{m}^{0}$. From eq. (18) we see that such a condition is fulfilled, under the conditions present between the neutrino-spheres, if $\sin ^{2} 2 \theta \lesssim 10^{-6}$.

The anisotropy in the total momentum of the escaping neutrinos can be computed as in [4, 8 ] starting from the resonance condition eq. (13). Since, as we discussed $Y_{e} \ll Y_{n}$ in the region of our interest, we neglect the second term on the right side of eq. (13). Following [4] we parametrize the resonance surface equation as follows

$$
r(\phi)=r_{0}+\delta \cos \phi
$$

where $r_{0}$ is the radius of the free-field resonance sphere defined by eq. (15). The displacement $\delta$ is found by subtracting the resonance condition computed for $\phi=\pi$ with the same computed in $\phi=-\pi$. We find

$$
Y_{e}(r+\delta)-Y_{e}(r-\delta) \simeq \frac{d Y_{e}}{d r} 2 \delta=2 \lambda_{e} Y_{e}\left(r_{0}\right) .
$$

Defining the $Y_{e}$ variation scale height by

$$
h_{Y_{e}} \equiv\left(\frac{d \ln Y_{e}}{d r}\right)^{-1}
$$

we can rewrite eq. (20) in the more compact form

$$
\delta=\lambda_{e} h_{Y_{e}}\left(r_{0}\right) .
$$

The deformation of the resonance sphere implies an angle dependence in the temperature of the escaping $\nu_{\tau}$ 's, hence to an asymmetry in the momentum that these neutrinos carry away from the SN. This is given by [8]

$$
\frac{\Delta k}{k} \approx \frac{1}{6} \frac{\int_{0}^{\pi} F_{\nu} \cos \phi \sin \phi d \phi}{\int_{0}^{\pi} F_{\nu} \sin \phi d \phi} \simeq \frac{2}{9} \delta h_{T}^{-1}
$$

where the temperature variation scale height $h_{T}$ is defined in analogy to the definition of $h_{Y_{e}}$ given in eq. (21). The factor $1 / 6$ in eq. (23) accounts for the fact that, out of the six neutrino and anti-neutrino species, we are assuming that only the $\nu_{\tau}$ carries a momentum anisotropy. We note, however, that an extra factor of two may be gained in eq. (23) if, at the same time, $\bar{\nu}_{\tau}$ emission suffers resonant conversion between $\bar{\nu}_{e}$ and $\bar{\nu}_{\tau}$ spheres. This is indeed possible as the resonance condition eq. (12) for 
$\nu_{e}-\nu_{\tau}$ and $\bar{\nu}_{e}-\bar{\nu}_{\tau}$ conversions coincide. This is a characteristic feature of this mechanism [12] which is in sharp contrast with the case of MSW conversions [5], where either neutrinos or anti-neutrinos can resonantly convert, but not both.

By substituting eq. (22) in eq. (23) we finally get

$$
\frac{\Delta k}{k} \approx \frac{2}{9} \frac{h_{Y_{e}}}{h_{T}} \lambda_{e}
$$

Numerical SN simulations [20] typically give $h_{Y_{e}} / h_{T} \approx 1$ between the two neutrino-spheres. Hence, we see from eq. (24) that in order for massless neutrino resonant conversions to account for the observed pulsar velocities one needs $\lambda_{e} \approx 5 \times 10^{-2}$ in the region between the two neutrino-spheres. Using the expression for the polarization $\lambda_{e}$ given in 21] we determine the required value of the magnetic fields strength as $\approx 10^{15}$ Gauss, which seems reasonable from the astrophysics point of view [22, and lower than required in the MSW [7] and RSFP [9] neutrino conversion mechanisms.

This is illustrated in Fig. 1 for three different choices of $\rho Y_{e}$ at resonance consistent with $\mathrm{SN}$ models. The three lines correspond to $\rho Y_{e}$ values (in g/c.c.) $5 \times 10^{9}$ (solid), $1 \times 10^{10}$ (dashed) and $5 \times 10^{10}$ (dotted) at resonance. In Fig. 1 we assume that both $\nu_{\tau}$ and $\bar{\nu}_{\tau}$ carry a momentum anisotropy. The kick velocity values given in the right ordinate assume a pulsar mass of $1.4 M_{\odot}$ and a total energy released by all neutrino species of $3 \times 10^{53} \mathrm{erg}$.

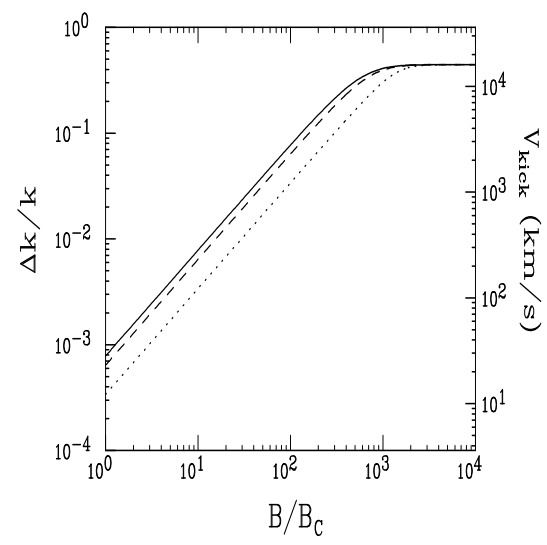

FIG. 1. Magnitude of kick velocity versus magnetic field, in units of the critical field $B_{c}=4.4 \times 10^{13}$ Gauss.

In short we have proposed a viable scheme for generating pulsar velocities from anisotropic neutrino emission induced by resonant conversions of massless neutrinos in the presence of a strong magnetic field. Neither neutrino masses nor magnetic moments are required and the parameters required are totally consistent with cosmology (e.g. nucleosynthesis) and astrophysics (e.g. supernova physics). However, our proposal rests on the idea that there is a small violation of universality in the weak interaction. Again, we are safely within the presently allowed region of parameters. However, this mechanism would become inconsistent should the sensitivity of weak universality tests improve significantly.

Acknowledgements: This work was supported by DGICYT grant PB95-1077, by a CICYT-INFN grant and by the TMR network grant ERBFMRXCT960090 of the European Union. H. N. was supported by a FAPESP. We thank A. Rossi for discussions.

[1] A.G. Lyne and D.R. Lorimer, Nature 369 (1994) 127.

[2] N.N. Chugai, Pis'ma Astron. Zh.10, 87, 1984.

[3] A. Vilenkin, Astrophys. J. 451 (1995) 700; for a recent reference see Dong Lai, Y.-Z. Qian, astro-ph/9712043

[4] A. Kusenko, G. Segrè, Phys. Rev. Lett. 77 (1996) 4872

[5] M. Mikheyev, A. Smirnov, Sov. J. Nucl. Phys. 42 (1986) 913; L. Wolfenstein, Phys. Rev. D17 (192369) 78; Phys. Rev. D20 (1979) 2634.

[6] J.C.D'Olivo, J.F.Nieves and P.B. Pal, Phys. Rev. D40 (1989) 3679; V.B. Semikoz, J.W.F. Valle, Nucl. Phys. B425 (1994) 65, err. Nucl. Phys. 485 (1997) 585; J.C.D'Olivo, J.F.Nieves, Phys. Lett. B383 (1996) 87; P. Elmfors, D. Grasso, G. Raffelt, Nucl. Phys. B479 (1996) 3

[7] A. Kusenko, G. Segrè, Phys. Rev. Lett. 79 (1997) 2751

[8] Y.Z. Qian, Phys. Rev. Lett. 79 (1997) 2750

[9] E.Kh. Akhmedov, A. Lanza and D.W. Sciama, Phys. Rev. D56 (1997) 6117

[10] E.Kh. Akhmedov, Phys. Lett. B213 (1988) 64-68; C. S. Lim and W. Marciano, Phys. Rev. D37 (1988) 1368

[11] J. Schechter, J.W.F. Valle, Phys. Rev. D24 (1981) 1883 and Phys. Rev. D25 (1982) 283

[12] J.W.F. Valle, Phys. Lett. B199 (1987) 432

[13] H. Nunokawa, Y.Z. Qian, A. Rossi and J.W.F. Valle, Phys. Rev. D54 (1996) 4356 [hep-ph/9605301]

[14] R. Mohapatra and J. W. F. Valle, Phys. Rev. D34 (1986) 1642; the same mass matrix was suggested in an extended left-right model by D. Wyler and L. Wolfenstein, Nucl. Phys. B218 (1983) 205.

[15] E. Witten, Nucl. Phys. B268 (1986) 79.

[16] See e.g., J. W. F. Valle, in Physics Beyond the Standard Model, hep-ph/9603307, published by the American Insitute of Physics and Prog. Part. Nucl. Phys. 26 (1991) 91 and Nucl. Phys. B (Proc. Suppl.) 11 (1989) 118

[17] J. Schechter and J. W. F. Valle, Phys. Rev. D22 (1980) 2227.

[18] M. Gronau, C. N. Leung, J. L. Rosner, Phys. Rev. D29 (1984) 2539; P. Langacker, D. London, Phys. Rev. D38 (1988) 886 and Phys. Rev. D38 (1988) 907; A. Ilakovac, A. Pilaftsis, Nucl. Phys. B437 (1995) 491; E. Nardi, E. Roulet, D. Tommasini, Phys. Lett. B344 (1995) 225.

[19] In eq. (10) we neglected the neutrino contribution to the potentials in between the two neutrino-spheres. 
[20] H. Susuki, private communication.

[21] H. Nunokawa, V.B. Semikoz, A.Yu. Smirnov and J.W.F. Valle, Nucl. Phys. B501 (1997) 17

[22] C. Thompson, R. Duncan, Astrophys. J. 408 (1993) 194 